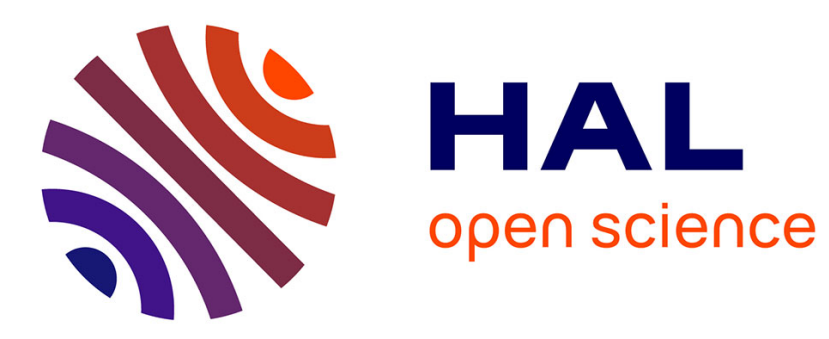

\title{
Your collective timeline of climate science history
}

Benoît Tournadre, Mélodie Trolliet

\section{To cite this version:}

Benoît Tournadre, Mélodie Trolliet. Your collective timeline of climate science history. EGU General Assembly 2020, May 2020, Vienne, Austria. 10.5194/egusphere-egu2020-3526 . hal-02949026

\section{HAL Id: hal-02949026 \\ https://hal.science/hal-02949026}

Submitted on 25 Sep 2020

HAL is a multi-disciplinary open access archive for the deposit and dissemination of scientific research documents, whether they are published or not. The documents may come from teaching and research institutions in France or abroad, or from public or private research centers.
L'archive ouverte pluridisciplinaire HAL, est destinée au dépôt et à la diffusion de documents scientifiques de niveau recherche, publiés ou non, émanant des établissements d'enseignement et de recherche français ou étrangers, des laboratoires publics ou privés. 


\section{A collective timeline of climate science history}

\section{PSL $\star$}

Maristech $\star$

\section{Centre O.I.E.}

Observation, Impacts, Energy (Sophia Antipolis, France)

AUTHORS

Benoît Tournadre (O.I.E)

Mélodie Trolliet (O.I.E)

PARTNERS

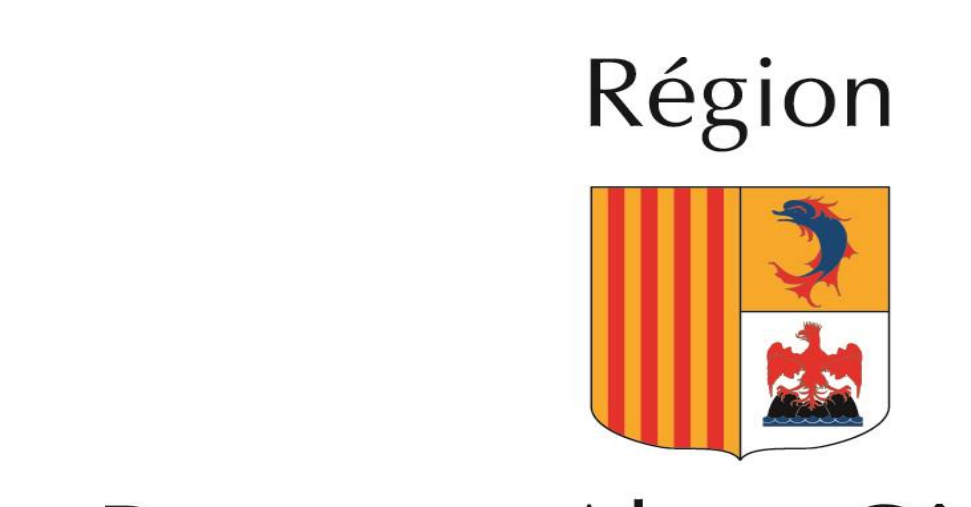

Provence-Alpes-Côte d'Azur

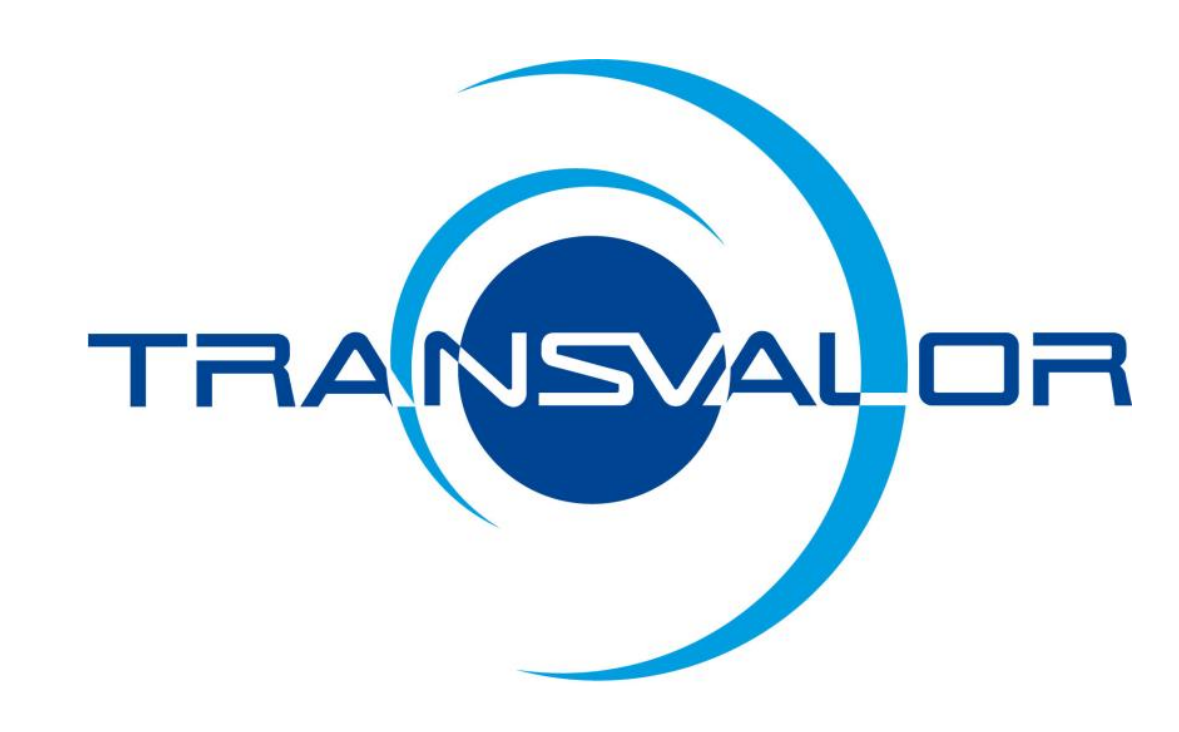

CONTACT

benoit.tournadre@mines-paristech.fr melodie.trolliet@mines-paristech.fr

www.sophia.mines-paristech.fr/earthbreath/ www.oie.mines-paristech.fr

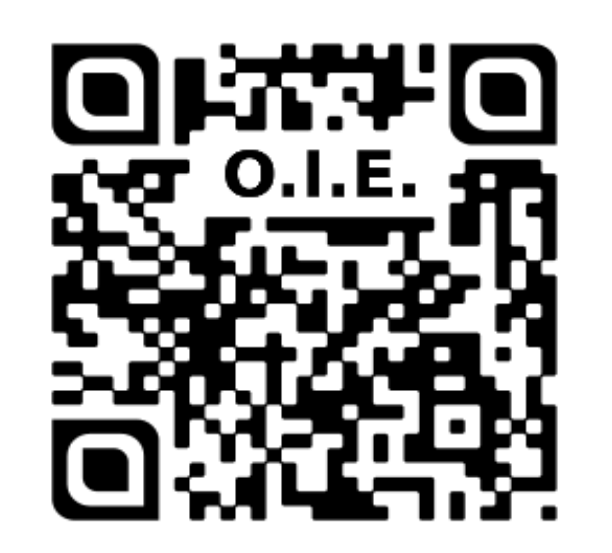

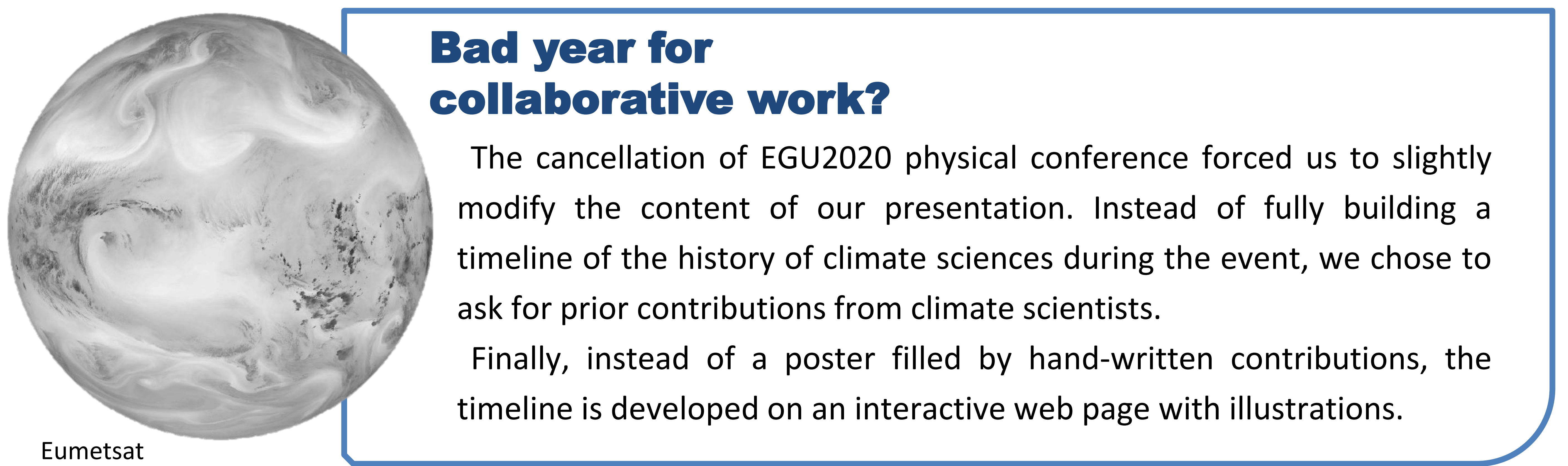

\section{Discover the interactive timeline online!}

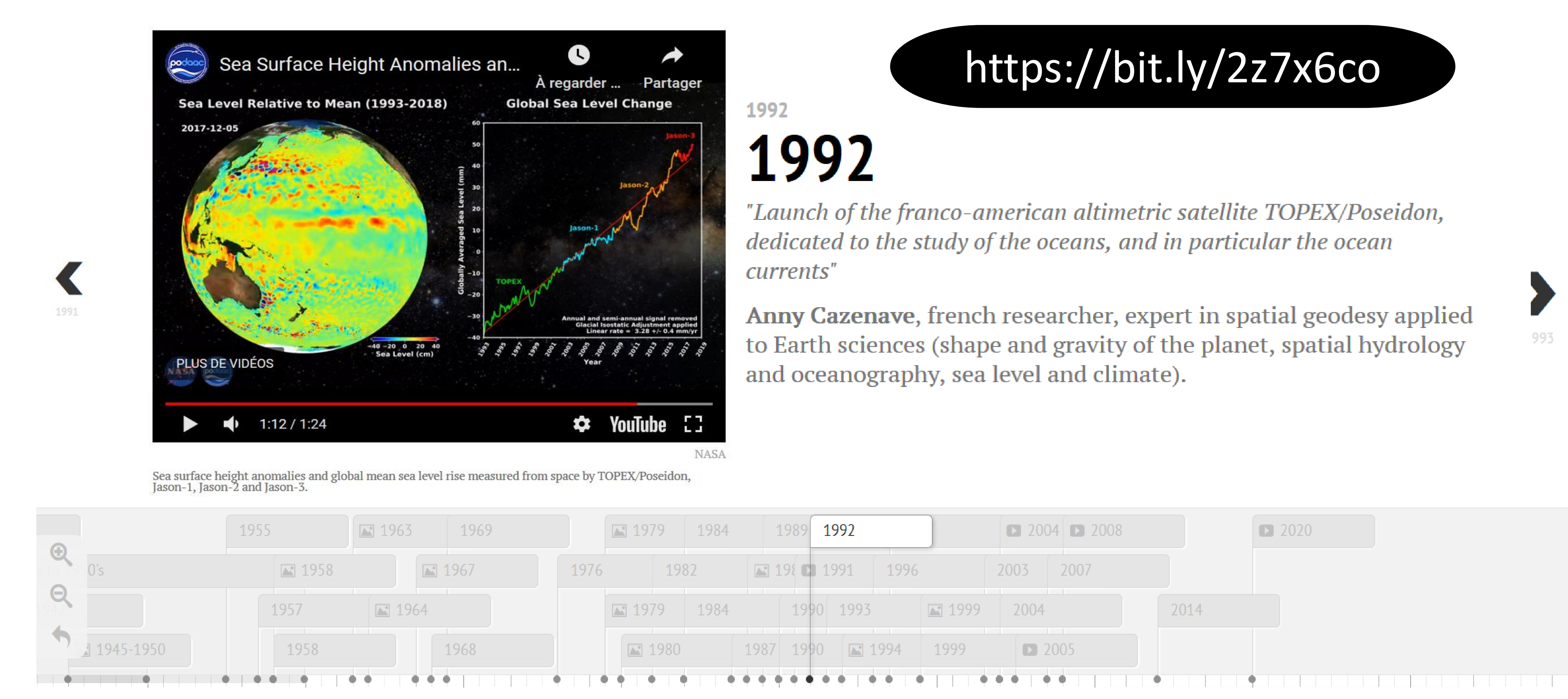

\section{First contributors}

We asked 47 scientists for their contributions: about 3 dates corresponding to what they consider as major breakthroughs in their respective areas of expertise.

At this point, 16 of them did answer.

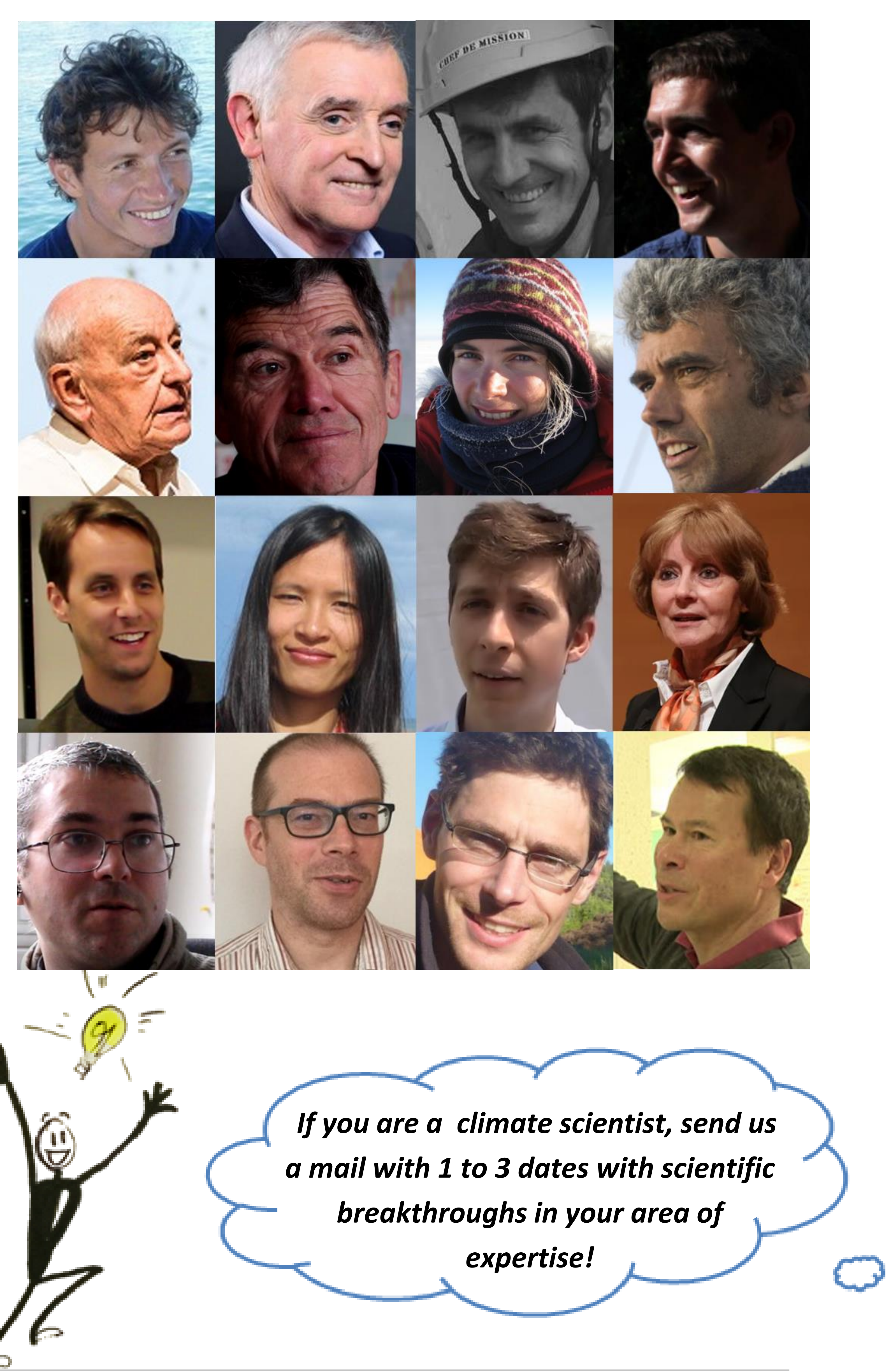

Find the timeline and more visual resources on our Earthbreath webpage And on Earthbreath Twitters: @eb climate data

$$
\text { @eb climat fr }
$$

$\rightarrow$ Glaciology

$\rightarrow$ Statistics of extreme events

$\rightarrow$ History of sciences

$\rightarrow$ Climate variability $\rightarrow$ Climate modeling
$\rightarrow$ Paleoclimatology

$\rightarrow$ Space oceanography $\rightarrow$ Ocean physics and geochemistry

$\rightarrow$ Atmosphere monitoring

$\rightarrow$ Spectroscopy

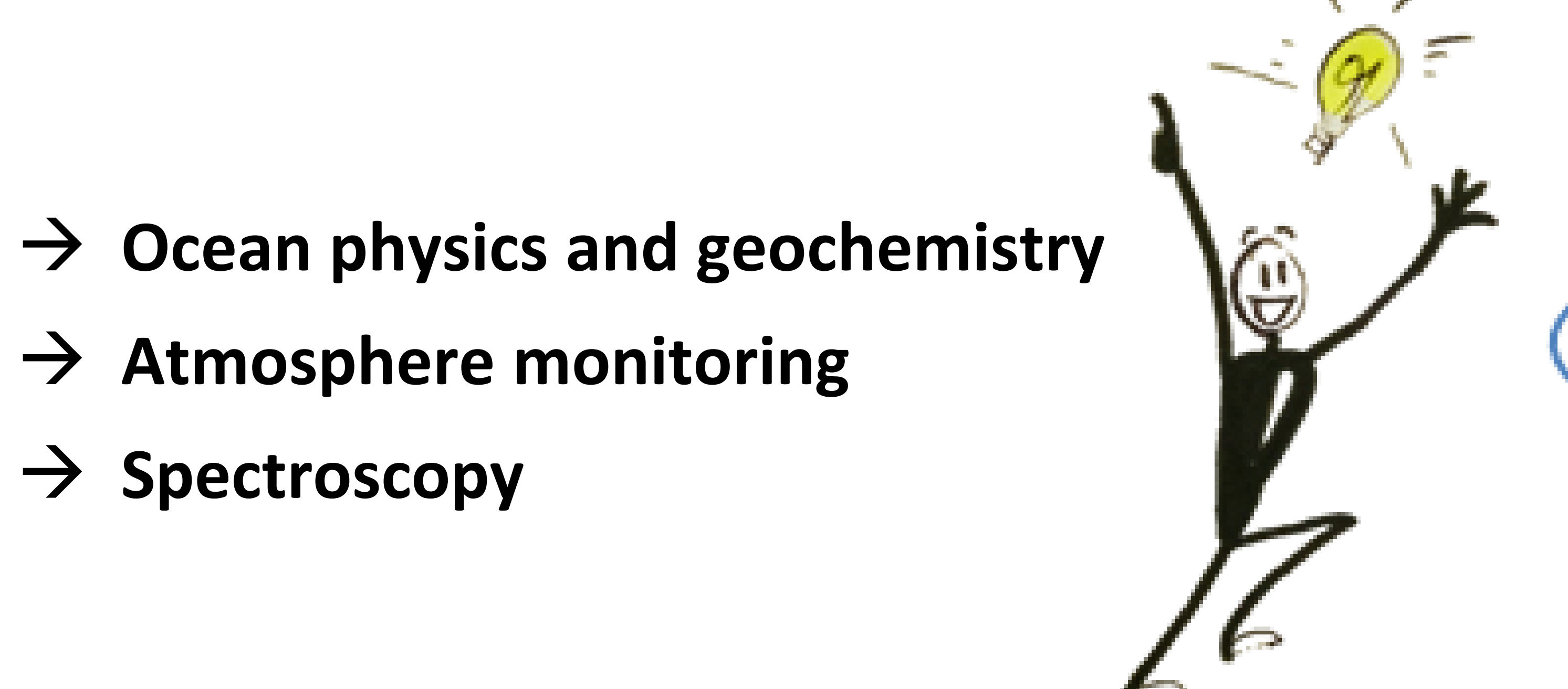

www.sophia.mines-paristech.fr/earthbreath 